\title{
Sensitivity of fetal RHD screening for safe guidance of targeted anti-D immunoglobulin prophylaxis: prospective cohort study of a nationwide programme in the Netherlands
}

\author{
Masja de Haas, ${ }^{1,2,3}$ Florentine F Thurik, ${ }^{1,4}$ Catharina P B van der Ploeg, ${ }^{5}$ Barbera Veldhuisen, ${ }^{1,3}$ \\ Hoang Hirschberg, ${ }^{6}$ Aicha Ait Soussan, ${ }^{1}$ Heleen Woortmeijer, ${ }^{3}$ Frithjofna Abbink, ${ }^{7}$ \\ Godelieve C M L Page-Christiaens, ${ }^{4}$ Peter G Scheffer, ${ }^{4}$ C Ellen van der Schoot ${ }^{1}$
}

For numbered affiliations see end of article.

Correspondence to: C E van der Schoot Department of Experimental

Immunohematology, Sanquin Research, PO Box 9190, 1006 AD Amsterdam, Netherlands e.vanderschoot@sanquin.n! Additional material is published online only. To view please visit the journal online.

Cite this as: BMJ 2016;355:i5789 http://dx.doi.org/10.1136/bmj.i5789

Accepted: 5 October 2016

\section{ABSTRACT}

OBJECTIVE

To determine the accuracy of non-invasive fetal testing for the $R H D$ gene in week 27 of pregnancy as part of an antenatal screening programme to restrict anti-D immunoglobulin use to women carrying a child positive for $R H D$.

\section{DESIGN}

Prospectively monitoring of fetal RHD testing accuracy compared with serological cord blood typing on introduction of the test. Fetal RHD testing was performed with a duplex real time quantitative polymerase chain reaction, with cell-free fetal DNA isolated from $1 \mathrm{~mL}$ of maternal plasma The study period was between 4 July 2011 and 7 October 2012. The proportion of women participating in screening was determined.

SETTING

Nationwide screening programme, the Netherlands. Tests are performed in a centralised setting.

PARTICIPANTS

25789 RhD negative pregnant women.

\section{MAIN OUTCOME MEASURES}

Sensitivity, specificity, false negative rate, and false positive rate of fetal RHD testing compared with serological cord blood typing; proportion of technical failures; and compliance to the screening programme.

RESULTS

A fetal RHD test result and serological cord blood result were available for 25789 pregnancies. Sensitivity for

\section{WHAT IS ALREADY KNOWN ON THIS TOPIC}

During pregnancy, fetal $R H D$ status can be determined by real time polymerase chain reaction using cell-free DNA isolated from maternal plasma

The reported sensitivity of fetal $R H D$ testing in the third trimester allows the use of this test to be explored for restricting antenatal anti-D immunoglobulin to RhD negative women carrying an RhD positive child

\section{WHAT THIS STUDY ADDS}

Fetal $R H D$ testing in week 27 of pregnancy is highly reliable and can be used as a single test to prevent unnecessary use of anti-D immunoglobulin during pregnancy in RhD negative women carrying an RhD negative child

Both antenatal and postnatal anti-D immunoglobulin prophylaxis can be administered based on this single test result without the need for additional cord blood testing

More than $98 \%$ of the women who participated in the antenatal screening programme participated in the fetal $R H D$ testing detection of fetal RHD was $99.94 \%$ (95\% confidence interval $99.89 \%$ to $99.97 \%$ ) and specificity was $97.74 \%$ (97.43\% to $98.02 \%)$. Nine false negative results for fetal $R H D$ testing were registered $(0.03 \%, 95 \%$ confidence interval $0.01 \%$ to $0.06 \%$ ). In two cases these were due to technical failures. False positive fetal $R H D$ testing results were registered for 225 samples (0.87\%, $0.76 \%$ to $0.99 \%)$. Weak RhD expression was shown in 22 of these cases, justifying anti-D immunoglobulin use. The negative and positive predictive values were $99.91 \%$ (95\% confidence interval $99.82 \%$ to $99.95 \%)$ and $98.60 \%$ (98.40\% to $98.77 \%$ ), respectively. More than $98 \%$ of the women participated in the screening programme.

\section{CONCLUSIONS}

Fetal $R H D$ testing in week 27 of pregnancy as part of a national antenatal screening programme is highly reliable and can be used to target both antenatal and postnatal anti-D immunoglobulin use.

\section{Introduction}

Haemolytic disease of the fetus and newborn caused by maternal alloantibodies against the RhD antigen of the rhesus system is potentially life threatening for the fetus. ${ }^{12}$ The introduction of postnatal anti-D immunoglobulin prophylaxis around 1970 has drastically decreased the risk of maternal alloimmunisation in $\mathrm{RhD}$ negative women carrying an $\mathrm{RhD}$ positive child, from $10-15 \%$ to $0.8-1.5 \% .^{3}$ Routine use of antenatal anti-D immunoglobulin in the late 1990s further reduced the risk of alloimmunisation to $0.18-0.35 \% .4-6$ The prevalence of RhD negativity varies between ethnic groups $(<1 \%$ in Chinese women, $5 \%$ in African black women, and $15 \%$ in white women), whereas the risk of carrying an RhD positive fetus for RhD negative women varies in these population from more than $99 \%$, to $80 \%$, and $60 \%$, respectively. ${ }^{7}$ In most women who are $\mathrm{RhD}$ negative there is a complete deletion of the $R H D$ gene. The discovery of cell-free fetal DNA in maternal plasma during pregnancy ${ }^{8}$ and the feasibility of fetal RHD testing with this source of DNA ${ }^{910}$ presented the opportunity to restrict antenatal anti-D immunoglobulin use to only those RhD negative women carrying an $\mathrm{RhD}$ positive child, which optimises the use of this blood product. ${ }^{11-14}$ The challenge of non-invasive fetal testing is the variable and low amount of fetal DNA present in maternal plasma. The challenge in fetal $R H D$ testing is further complicated by the genetic variation of $R H D$ alleles, present in the fetus or mother. ${ }^{15}$ Examples are the $R H D^{*} 01 N .01\left(R H D^{*} \Psi\right)$ gene, with a high 
prevalence in $\mathrm{RhD}$ negative women in the black population, and the $R H D^{\star} 06\left(R H D^{*} D V I\right)$ gene in white women. In women carrying these genes, positive polymerase chain reaction (PCR) test results could be obtained from maternal $R H D$ sequences. To make fetal $R H D$ testing possible in carriers of these genes, sequences that are present in normal and variant $R H D$ genes (ie, $R H D$ exon 7) as well as sequences that are present in normal $R H D$ genes but absent in mothers carrying one of the most common $R H D$ variant alleles (ie, $R H D$ exon 5) have to be included in the design of PCR tests. ${ }^{16}$ Theoretically, with such a design, false positive results can be prevented and the fetal $R H D$ genotype can be based on the amplification of $R H D$ exon 5. This enables fetal $R H D$ testing in a multiethnic population.

The first large scale introduction of non-invasive fetal RHD testing and targeted antenatal anti-D immunoglobulin use was in 2010 in Denmark. ${ }^{17}$ In the Netherlands, a feasibility and cost effectiveness study led the Dutch Health Council to recommend testing. ${ }^{18}$ Subsequently, the Ministry of Health sanctioned the introduction of fetal RHD testing in week 27 of pregnancy by the national antenatal screening programme. It was started in July 2011 and aimed to restrict use of both antenatal and postnatal anti-D immunoglobulin use to those RhD negative pregnant women with fetuses that tested positive for RHD. In addition, the Ministry of Health commissioned an evaluation study to judge the logistics of centralised testing and fetal $R H D$ test performance. The hypothesis was that this centralised testing would result in less than $0.25 \%$ false negative test results, which would allow the omission of cord blood testing. We report the performance of fetal RHD testing for test accuracy and compliance to the Dutch screening programme, as evaluated in the first 15 months after introduction, encompassing more than $32000 \mathrm{RhD}$ negative pregnant women.

\section{Methods}

Fetal RHD testing is part of the antenatal screening programme for infectious diseases and red blood cell immunisation, offered to all pregnant women in the Netherlands (around 180000 each year). This antenatal screening programme is organised by the National Institute for Public Health and the Environment (RIVM) on behalf of the Minister of Health, Welfare and Sport and funded by government resources. The RIVM records and stores personal and laboratory data for all pregnant women in a central database (Praeventis) from the first antenatal visit onwards. The first antenatal visit includes RhD typing and red blood cell antibody screening at a local laboratory, generally before week 13 of pregnancy. If a weak D expression is observed, the presence of weak D type 1, 2, or 3 should be investigated and these women are regarded as RhD positive and not at risk for alloimmunisation. Subsequently, fetal $R H D$ testing and repeated red blood cell antibody screening is offered in week 27 to all non-RhD immunised RhD negative pregnant women. The blood tests in week 27 are performed by Sanquin Diagnostic Services in Amsterdam.

\section{Blood samples}

On request of the woman's obstetric care provider (midwife, general practitioner, or gynaecologist) $9 \mathrm{~mL}$ of EDTA anticoagulated blood is drawn between 27 and 29 weeks of gestation at a local laboratory. This blood sample and cord blood samples taken at delivery are transported to Sanquin either by surface mail or by Sanquin's courier service. Fetal RHD typing was performed during pregnancy, thus before serology testing of cord blood.

\section{Serological typing and antibody screening}

$\mathrm{ABO}$ and RhD typing and antibody screening was performed with the automated WA-Diana system (DiaMed, Cressier, Switzerland) or AutoVue Innova system (Ortho Clinical Diagnostics, Raritan, NJ) following the manufacturer's protocols. In accordance with the Dutch guidelines, RhD typing is performed with an anti-D reagent (LHM 59/20 [LDM3]+175-2) that does not recognise the RhD category VI (DVI) phenotype, since DVI carriers can become RhD alloimmunised and prophylaxis is therefore indicated, whereas cord blood serology was performed with anti-D reagents (LHM 59/20 [LDM3]+175-2 and ESD-1M+175-2) that do detect DVI, since red blood cells with this phenotype might lead to alloimmunisation. We further investigated positive results of serology with $2+$ or less reaction strength manually using in-house available reagents with the indirect antiglobulin test and molecular testing with the multiplex ligation dependent probe amplification (MLPA) assay or RHD exon specific sequencing to determine the type of variation in $R H D$, as described previously. ${ }^{19}$

\section{Automated plasma separation and DNA extraction}

Plasma was collected before any other handling of the sample. Blood samples were centrifuged at $1200 \times \mathrm{g}$ for 10 minutes and $2.5 \mathrm{~mL}$ of plasma was robotically dispensed into $5 \mathrm{~mL}$ tubes using a Xiril robotic workstation (Xiril, Hombrechtikon, Switzerland). The remains of the blood sample were used for antibody screening and maternal serology. The plasma fraction was centrifuged at $2400 \times \mathrm{g}$ for 20 minutes and subsequently dispensed into two 96 well plates with the Xiril robot, $1 \mathrm{~mL}$ of plasma in each plate. One plate was stored at $-20^{\circ} \mathrm{C}$ as a backup; the other was presented to the MagNa Pure 96 Instrument (Roche Holding, Basel, Switzerland) for automated DNA extraction (Viral NA Large Volume Kit; Roche), with a final elution volume of $50 \mu \mathrm{L}$.

\section{Real time $P C R$ analysis}

We carried out duplex real time polymerase chain reaction analysis for RHD exon 5 and $R H D$ exon 7 in triplicate on cell-free DNA isolated from maternal plasma, generating six test results for each blood sample. ${ }^{1620}$ The RHD exon 5 PCR does not amplify RHD*01N.01 $\left(\mathrm{RHD}^{\star} \Psi\right)$ and $R H D^{\star} 06$ (RHD $\left.{ }^{\star} \mathrm{DVI}\right)$. All PCR tests were performed with the StepOnePlus Real-Time PCR System (Applied Biosystems, Foster City, CA).

The reactions were set up in a final volume of 25.35 $\mu \mathrm{L}$, with $15 \mu \mathrm{L}$ of extracted DNA (Xiril Gamma robotic workstation; Xiril, Hombrechtikon, Switzerland). 
In the first 10400 samples, PCR conditions were 20 seconds at $95^{\circ} \mathrm{C}$, followed by 50 cycles of one second at $95^{\circ} \mathrm{C}$ and 20 seconds at $60^{\circ} \mathrm{C}$ performed with Taqman Fast Universal PCR Master Mix, No AmpErase UNG (Applied Biosystems) ("fast protocol”). We collected cycle threshold values at fixed thresholds of 0.05 . Because of variation in the performance of PCR tests such as large dispersion in the delta Rn, especially for $R H D$ exon 7, or a delta $\mathrm{Rn}$ that did not exceed 1.0, we transitioned to two minutes at $50^{\circ} \mathrm{C}, 10$ minutes at $95^{\circ} \mathrm{C}$, followed by 50 cycles of 15 seconds at $95^{\circ} \mathrm{C}$, and one minute at $60^{\circ} \mathrm{C}$ performed with Taqman Universal PCR Master Mix, No AmpErase UNG (Applied Biosystems) ("slow protocol”). In each run of 48 samples, we used an internal standard (pooled plasma) for quality purposes. All samples were tested within one working day. Up to three runs were performed each day. PCR performance of a run was considered valid to meet the quality threshold if the cycle threshold values of the internal standard were 40.0 or less in at least two of three replicates.

For each sample, we performed PCRs in triplicate. A prediction algorithm was used for automated scoring by computer (see supplementary eTable 1 and eTable 2). Each of the six amplification signals was considered and automatically scored as representing maternal DNA (ie, cycle threshold values in the range of maternal DNA levels), fetal DNA, non-specific (ie, non-specific amplification signals or low level of fetal DNA), or negative (ie, no amplification after 50 cycles) (see supplementary eTable 1). Based on the combination of scored cycle threshold values, the computer algorithm provided the following conclusions, respectively: "fetus RhD positive," "fetus RhD negative," or "no result" combined with or without advice to repeat the test. The scoring algorithm is shown in supplementary eTable 2, including the number of samples for each combination of cycle threshold values and the corresponding results for cord blood serology. A supervisor assessed the computed results daily and the protocol allowed for manual over-ruling based on the visual inspection of the amplification plots.

In all cases in which a fetal or maternal $R H D$ variant was suspected, we reported the result as fetus positive and stored the material for research purposes. During the study period we changed the preset prediction algorithm of computer software once, after about 7700 samples (see supplementary eTable 1) when we changed from a fast to slow PCR protocol. Reports were automatically generated and electronically transferred to both the pregnant woman's care provider and the nationwide registration database of the RIVM. If a fetal RhD positive test result was issued, care providers were advised to administer $200 \mu \mathrm{g}$ (1000 IU) of anti-D immunoglobulin in the 30th week of gestation as well as within 48 hours after birth. If a fetal RhD negative test result was issued, it was reported that use of anti-D immunoglobulin was considered unnecessary. Thus since the launch of the screening programme the anti-D immunoglobulin prophylaxis policy was entirely based on the fetal RHD test result and not on cord blood serology. Fetal $R H D$ testing was repeated manually on the stored plasma samples of the false negative cases identified by cord blood serology, and PCRs for extra fetal markers were performed as described previously. ${ }^{21}$

\section{Cord blood analysis}

As part of the national evaluation of the fetal $R H D$ screening programme, samples of postnatal cord blood were sent to our laboratory to determine the newborn's $\mathrm{RhD}$ serology. Cord blood serology was used as reference standard since it was considered the best test available for determination of neonatal $\mathrm{RhD}$ status and it was clinical practice before the launch of the fetal RHD screening programme. Cord blood testing was performed and interpreted without knowledge of the fetal RHD test results and was performed with the WA-Diana system (DiaMed) using two monoclonal anti-D reagents, LHM 59/20 (LDM3)+175-2 and ESD-1M+175-2. The latter recognises the DVI phenotype, which is regarded as immunogenic. Serology tests were performed on all days, except Sunday and Bank Holidays. All cord blood serology results were immediately compared with the fetal $R H D$ test result. Putative false negative fetal $R H D$ test results were immediately reported to the obstetric caregiver for timely use of postnatal anti-D immunoglobulin.

\section{Molecular characterisation of RHD variant genes}

We scored all samples with positive $R H D$ amplifications as fetal $R H D$ positive according to the scoring algorithm, and all weak or variable cord blood serological results as serologically $\mathrm{RhD}$ positive. However, all maternal or newborn samples in which the fetal $R H D$ PCR test or the cord blood serology suggested the presence of an $R H D$ variant, were comprehensively analysed for research purposes. The results of these analyses have been published elsewhere ${ }^{22}$ but were not used for the scoring algorithms.

\section{Selection of study population}

All serologically $\mathrm{RhD}$ negative pregnant women with a request for a fetal $R H D$ typing test at Sanquin Blood Supply were eligible. We included only women with a risk for maternal anti-D alloimmunisation. $\mathrm{RhD}$ positive women were excluded. Women were included when the fetal RHD test was performed between 4 July 2011 and 7 October 2012 and thus formed a consecutive series. Blood samples were not tested when the blood was drawn before the 27 th gestational week or when the transfer time of the samples exceeded five days. Additionally, we excluded women with anti-red blood cell antibodies and those with multiple pregnancies.

\section{Data collection and analysis}

We prospectively collected data on all fetal RHD tests performed in the first 15 months of the national screening programme (4 July 2011 to 7 October 2012) and cord blood tests until 31 December 2012 directly from our laboratory database. Statistical analyses were performed using GraphPad Prism version 5.01 for Windows and software from www.medcalc.org. For fractions close to boundary values of 0 or 1 we calculated $95 \%$ confidence intervals using a correction by Fleiss. ${ }^{23}$ 
The performance of the fetal RHD test was monitored every four weeks (independent of sample size) to enable adaptations to the antenatal screening programme if the false negativity rate would exceed the preset limit of $0.25 \%$. Compliance to fetal $R H D$ testing was also calculated every four weeks for one year after implementation, using the data from the central database Praeventis. We calculated compliance for women with an expected date of delivery between 30 September 2011 and 28 September 2012, corresponding to the 27 weeks' gestation mark occurring between 1 July 2011 and 29 June 2012. For samples from 112 women without a registered fetal $R H D$ test result and with an expected date of delivery in January or February 2012, we contacted the obstetric care provider to ask for information on the decision not to perform fetal $R H D$ testing.

Data were available for the following descriptive variables: maternal age, ethnicity, parity, gestational age at time of sampling, and cell-free fetal DNA level (table 1). The variables maternal age and cell-free fetal DNA level were available in the fetal $R H D$ test dataset. The fetal $R H D$ test dataset was linked to clinical data of the perinatal registry in the Netherlands for the purposes of a different study. This was done only for pregnancies with a cord blood serology result. We extracted data on ethnicity, parity, and gestational age at time of blood sampling from this linked and anonymised dataset.

\section{Patient involvement}

No patients were involved in setting the research question or the outcome measures, nor were they involved in developing plans for recruitment, design, or implementation of the study. No patients were asked to advise on interpretation or writing up of results. There are no plans to disseminate the results of the research to study participants or the relevant patient community.

\section{Results}

\section{Study population}

Within the study period, 32622 blood samples from pregnant women reported to be $\mathrm{RhD}$ negative were sent to our laboratory for fetal $R H D$ testing as part of the

\begin{tabular}{|c|c|c|}
\hline Characteristics & Distribution & Data available \\
\hline Mean (SD) maternal age (years)* & $30.8(4.8)$ & $32160(100.0)$ \\
\hline Ethnicity (No (\%)): & & $21536(67.0)$ \\
\hline European* & $19478(90.4)$ & \\
\hline Mediterraneant & $879(4.1)$ & \\
\hline Black Creoleł & $168(0.8)$ & \\
\hline Asian§ & $85(0.4)$ & \\
\hline Hindustani & $66(0.3)$ & \\
\hline Other & $860(4.0)$ & \\
\hline Parity (No (\%)): & & $21579(67.1)$ \\
\hline Nulliparous & $9712(45.0)$ & \\
\hline Multiparous & $11867(55.0)$ & \\
\hline Mean (SD) gestational age at sampling (weeks+days) & $27^{6}\left(0^{6}\right)$ & $21579(67.1)$ \\
\hline Median (range) cell-free fetal DNA level (genome equivalents $/ \mathrm{mL})^{c}$ & $46.6(1.5-868.5)$ & $13076(40.7)$ \\
\hline $\begin{array}{l}\text { *Not including Mediterranean countries. } \\
\text { †All countries surrounding the Mediterranean Sea. } \\
\text { ‡Black Creole includes all black people. } \\
\S \text { Asian included all Asian countries, with the exception of people of Hinc }\end{array}$ & tani descent. & \\
\hline
\end{tabular}

screening programme (fig 1 ). Because of administrative errors, 382 samples were from RhD positive women (1.17\%). For 18 samples, serological RhD testing of the pregnant woman showed weak $(\leq 2+)$ reactivity, and further analysis showed that these women carried an $R H D$ variant allele, hampering fetal testing in most cases. After exclusion of these 400 cases, fetal RHD testing was performed for 32222 pregnancies. A total of 62 women were pregnant twice during the study period. Table 1 shows the characteristics of the population.

\section{Technical performance of the fetal $R H D$ assay}

A total of 883 DNA isolation runs were performed and 21 (2.4\%) were repeated owing to either technical failure of the DNA isolation robot $(n=7)$ or failure to meet the quality threshold defined by the internal standard ( $\mathrm{n}=14$; only samples with a negative $R H D$ test result were repeated). In 19862 cases (61.6\%) an $\mathrm{RhD}$ positive test result and in 12360 cases (38.4\%) an $\mathrm{RhD}$ negative test result were obtained and reported. In 356 cases $(1.1 \%)$ the results were issued after the assay had been repeated, either by indication of the software $(n=190)$ or by manual decision of the supervisor $(n=166)$. In 25 cases $(0.08 \%)$ the software was over-ruled and the result was reported as $\mathrm{RhD}$ positive without the test being repeated. In five cases a new blood sample was requested.

In general, there was a clear discrimination between positive and negative $R H D$ test results: in $99.3 \%$ of the negative results no or a single amplification signal was obtained, and in $98.4 \%$ of the positive results five or six positive signals were obtained (see supplementary eTable 2). The scoring algorithm aims to prevent false negative results, therefore a positive result was issued in all cases with only three positive amplification signals ( $n=233 ; 0.72 \%)$. The variance in mean cycle threshold values between different samples was relatively large (see supplementary eFigure 1). In 95\% of the plasma samples the amount of isolated fetal DNA varied between 8 and 142 genome equivalent (geq) per millilitre, with a median concentration of 40 geq $/ \mathrm{mL}$.

We received cord blood samples from 25789 out of the 32222 pregnancies (80\%). We received slightly fewer cord blood samples in the group with a fetal RHD negative result ( $40.6 \%$ of cases without a cord blood sample versus $37.8 \%$ of cases with a cord blood sample had an RHD negative test result, $\mathrm{P}<0.001$.

\section{Diagnostic accuracy of the fetal $R H D$ assay}

Fetal $R H D$ testing was negative for nine pregnancies, whereas cord blood serology showed that the newborn was RhD positive (fig 1 and table 2). In 225 cases the fetus had been reported as $R H D$ positive, whereas cord blood serology showed the newborn to be RhD negative (fig 1). Overall, the negative predictive value was 99.91\% ( $95 \%$ confidence interval $99.82 \%$ to $99.95 \%$ ) and the positive predictive value was $98.60 \%$ (98.40\% to $98.77 \%$ ). Supplementary eTable 3 provides the false negative and false positive rates by ethnic group. The sensitivity for detection of fetal RHD was $99.94 \%$ (99.89\% to 99.97\%) and specificity was $97.74 \%$ (97.43\% to $98.02 \%$ ). 
Extensive analysis was performed on the nine false negative cases (see supplementary eTable 4). Genotyping on the cord blood showed the presence of a normal RHD gene in these neonates. Furthermore, DNA fingerprinting performed with the stored maternal whole blood sample, the back-up plasma sample, and the cord blood sample ruled out sample mix-up (data not shown). In retrospect, the PCR carried out on one of these samples (unique case number (UCN) 3, showed a suboptimal fluorescent signal, but the cycle threshold values obtained with internal standards were within the preset limits. In another case (UCN4), the operator had ignored a failure of the robot pipetting the plasma. In UCN6 the repeated assay showed normal fetal RHD signals suggestive of an unnoticed technical failure. In the other six cases either no fetal DNA (UCN1) or very low concentrations of fetal DNA were found on repeating the test with stored plasma.

Compared with routine cord blood serology, there were 225 false positive results, hence PCR assay during pregnancy indicating an $R H D$ positive fetus and cord blood serology indicating an $\mathrm{RhD}$ negative fetus (fig 1 and table 2). In 10 cases an incorrect blood sample had been sent in after delivery; in nine cases we confirmed this to be maternal blood or a mixture of maternal and cord blood, and in one case a sample mix-up had occurred. In 100 of the remaining 215 samples (46.5\%) a variant $R H D$ gene was detected in the mother and in the

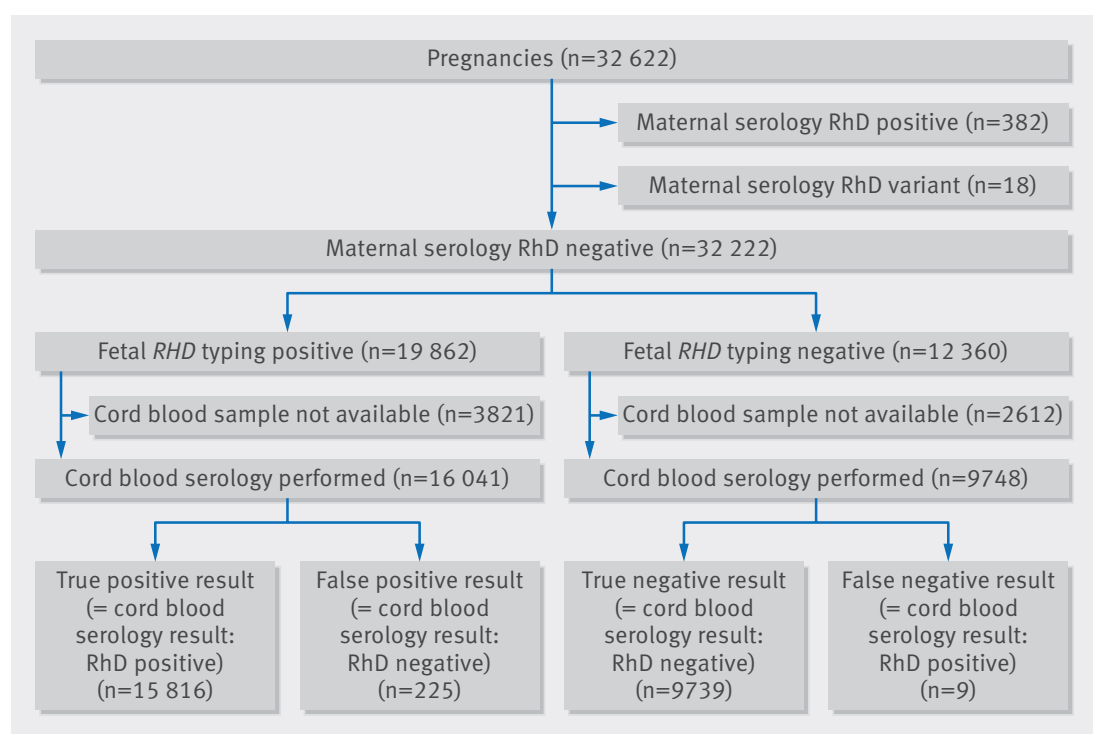

Fig 1 | Overview of reported fetal $R H D$ test results compared with postnatal cord blood serology results. Concurrence was $99.09 \%$ (25555/25789, $95 \%$ confidence interval $98.97 \%$ to $99.20 \%)$

Table 2 Cross tabulation of fetal RHD test results (index test) and cord blood serology results (reference standard)

\begin{tabular}{lllll} 
& \multicolumn{2}{l}{$\begin{array}{l}\text { Cord blood serology result } \\
\text { (reference standard) }\end{array}$} & \\
\cline { 2 - 3 } Fetal RHD test result (index test) & No positive & No negative & & Total No \\
\hline Positive & 15816 & 225 & 16041 \\
\hline Negative & 9 & 9739 & 9748 \\
\hline Total & 15825 & 9964 & 25789 \\
\hline
\end{tabular}

newborn $(n=55)$ or only in the newborn $(n=45)$; hence, the PCR results were correct. For 22 of these 45 newborns, follow-up serology or molecular testing showed the presence of a variant $R H D$ gene that produced $\mathrm{RhD}$ positivity of the red blood cells. Thus, in these latter cases the result of routine cord blood serology was false negative. For all the cases in which a variant $R H D$ gene was found in a serologically $\mathrm{RhD}$ negative typed mother, this had already been suspected owing to low cycle threshold values, representing high concentrations of $R H D$ sequences in the plasma. In the remaining 115 cases, 71 had false positive fetal $R H D$ test results owing to our strict scoring algorithm, which mainly aimed to prevent false negative cases: high cycle threshold values were found with three or less positive amplification signals $(n=44)$ or only four positive amplification signals $(n=27)$. In the remaining 44 cases there were five or six positive amplification signals (discussed in detail elsewhere ${ }^{24}$ ).

\section{Compliance to the fetal $R H D$ screening programme}

To determine compliance to the fetal $R H D$ screening programme, for all $\mathrm{RhD}$ negative women registered in the central database Praeventis ( $n=24986)$, we evaluated whether fetal $R H D$ testing had been requested and performed. Overall compliance was $96.3 \%$ for the first year after implementation, ranging from $91.1 \%$ in the first four weeks' period to around $97.5 \%$ towards the end of the study period. Four to five months after implementation (October-November 2011), we investigated why fetal RHD testing had not been performed in 112 cases (table 3). In 69 of these 112 cases (representing 1.8\% of the RhD negative women, as calculated after extrapolation), there was a justifiable reason, such as moving abroad $(n=31)$ or loss of pregnancy or delivery before week $27(\mathrm{n}=12)$. In 43 cases $(1.2 \%)$ the test was not requested, either mistakenly or for unknown reasons. As over $99 \%$ of all $\mathrm{RhD}$ negative pregnant women are registered in Praeventis, ${ }^{25}$ we conclude that participation in fetal $R H D$ testing as part of the nationwide screening programme was at least $98 \%$.

\section{Discussion}

In this paper we demonstrate that the accuracy of a fetal RHD assay, performed at week 27 of pregnancy as part of an antenatal screening programme, using a fully automated assay with a complete electronic sample tracking, result interpretation, report generation, and data transfer to minimise administrative errors is highly accurate. This accuracy allows the use of this test in the Netherlands to restrict the use of both antenatal and postnatal anti-D immunoglobulin to RhD negative pregnant women who carry an RhD positive child and are therefore at risk for $\mathrm{RhD}$ alloimmunisation. During a 15 month period, blood samples from $32222 \mathrm{RhD}$ negative women were tested, representing at least $98 \%$ of the RhD negative pregnant population. In $80 \%$ of cases, cord blood samples $(n=25789)$ were received to assess test performance. Cord blood serology showed nine false negative fetal $R H D$ test results $(0.03 \%$, 95\% confidence interval $0.02 \%$ to $0.07 \%$ ) and 225 false positive 


\begin{tabular}{|c|c|}
\hline Reasons for missing results & No (\%) \\
\hline Test not performed, woman did not qualify for programme: & $69(1.8)$ \\
\hline Moving abroad & $31(0.8)$ \\
\hline Week 12 blood sample test, but not pregnant & $7(0.2)$ \\
\hline Incorrect registration as RhD negative & $4(0.1)$ \\
\hline Formation of maternal anti-D antibodies & $5(0.1)$ \\
\hline Loss of pregnancy $<27$ weeks & $4(0.1)$ \\
\hline Delivery $<27$ weeks & $8(0.2)$ \\
\hline First consultation $>27$ weeks & $2(0.1)$ \\
\hline Refusal, father RhD negative & $6(0.2)$ \\
\hline Refusal, eg religious beliefs & $2(0.1)$ \\
\hline Test not performed, reason unknown: & $41(1.1)$ \\
\hline Test not requested by obstetric care provider & $29(0.8)$ \\
\hline No follow-up obstetric care provider & $12(0.3)$ \\
\hline Test not performed, other reason: & $2(0.1)$ \\
\hline Insufficient quantity blood sample, no repeat sample sent & $2(0.1)$ \\
\hline Total & $112(3.0)$ \\
\hline
\end{tabular}

fetal $R H D$ test results $(0.87 \%, 0.76 \%$ to $1.00 \%)$. In false negative cases preventive anti-D immunoglobulin was wrongly withheld while there was a risk of maternal anti-D immunisation. This could have resulted in maternal anti-D immunisation and eventually in haemolytic disease of the fetus and newborn in the current or subsequent pregnancy. In false positive cases anti-D immunoglobulin was wrongly administered as there was no risk of maternal anti-D immunisation. In these cases a scarce and expensive blood product is thus wasted, but no serious health risks are involved. Since we observed that unexpected technical failures can occur, we now implement a non-human sequence into the assay as an internal control for DNA isolation to further reduce the false negative rate. To secure the high quality of the Dutch antenatal screening programme, a prerequisite of the performance of fetal $R H D$ testing was that the false negative rate would not exceed that of cord blood serology, which was estimated to be $0.25 \%$ on the basis of previous work. ${ }^{426}$ Also, in our series with optimal administrative procedures, we identified at least 10 incorrectly assigned blood samples, labelled as cord blood; in nine cases we confirmed these to be from the mother (failure rate of $0.06 \%$ (10/16041; 95\% confidence interval $0.03 \%$ to $0.12 \%$ ).

Additional serological and molecular testing of cord blood samples typed as RhD negative when the results of fetal RHD testing was positive showed that in $0.09 \%$ (22/25789; $0.06 \%$ to $0.14 \%$ ) cord blood serology was in fact false negative. In these cases, anti-D immunoglobulin use on the basis of fetal RHD testing might have prevented $\mathrm{RhD}$ alloimmunisation, although in these cases the low RhD expression of the fetal red blood cells might only entail a low risk of alloimmunisation.

To minimise the number of inconclusive results, fetal $\mathrm{RhD}$ positive results were issued if any $R H D$ sequences were detected in maternal plasma. Positive results were also issued in those cases in which a pregnant women was suspected of carrying an $R H D$ variant allele hampering fetal $R H D$ testing. In accordance with other fetal RHD testing programmes, ${ }^{1427}$ we currently issue an inconclusive fetal $R H D$ test result when there are only positive amplifications suggestive of the presence of an $R H D$ variant gene in the mother: thus, in mothers carrying the rare $\mathrm{D}$ negative variant genes from which both exon 5 and exon 7 are amplified $(0.37 \%$ of Dutch pregnant women $)^{22}$ and in mothers carrying the more common $R H D^{*} 01 N .01\left(R H D^{*} \Psi\right)$ gene $(0.45 \%)^{22}$ or $R H D^{*} 06\left(R H D^{*} D V I\right)$ gene $(0.16 \%)^{22}$ from which exon 7 is amplified and in whom no fetal signal from exon 5 is observed. In these cases, we advise the use of antenatal anti-D immunoglobulin prophylaxis and cord blood serology to guide postnatal anti-D immunoglobulin use. In all women with the most commonly occurring $R H D$ variants $R H D^{*} \Psi$ and $R H D^{*} D V I$ in whom an exon 5 amplification is observed, a positive test result can be issued and no additional cord blood serology is needed. Using the new algorithm a false positive result would have been prevented in $0.21 \%$ (55/25789; $95 \%$ confidence interval $0.16 \%$ to $0.28 \%$ ) of women, at the expense of $0.51 \%$ (all women with a variant gene and not a fetal exon 5 amplification signal) inconclusive results. In these cases, we advise the use of antenatal anti-D immunoglobulin prophylaxis and to administer postnatal anti-D immunoglobulin on the basis of the cord blood serology result. Taking into account this inconclusive category, the false negative cord blood results and the cases for which no correct cord blood sample was received, the percentage of false positive test results was $0.57 \%$ (225 minus 55 and 22, respectively, and excluding the 10 incorrect cord blood samples, thus $148 / 25779$; $95 \%$ confidence interval $0.49 \%$ to $0.68 \%$ ). In these cases, anti-D immunoglobulin was unnecessarily administered.

\section{Comparison with other studies}

In our study the false negative rate for fetal $R H D$ testing of $0.03 \%$ (95\% confidence interval $0.02 \%$ to $0.07 \%$ ) has not been determined in such a large sample size previously but has been reported to range from $0.1 \%$ to $0.2 \%$ in weeks $11-13$ of pregnancy, ${ }^{28} 29$ and was $0.087 \%$ from week 24 onwards. ${ }^{27}$ The rate of false positive results obtained in our design of fetal RHD testing of $0.57 \%$ (95\% confidence interval $0.49 \%$ to $0.68 \%$ ) is comparable to other studies carrying out tests after week 24 of pregnancy (eg, $0.32 \%{ }^{26}$ and $0.8 \%{ }^{14}$ ), whereas our rate for inconclusive results of $0.21 \%$ is far lower than the published figures of $2.2 \%{ }^{27}$ and $3.2 \% .^{14}$ This confirms that our design of fetal RHD testing is suitable for testing in a multiethnic population.

For the Dutch screening programme, pre-implementation calculations had indicated that the introduction of fetal $R H D$ testing resulting in a $24 \%$ reduction in usage of anti-D immunoglobulin, and concomitant discontinuation of cord blood testing, would not increase the costs of the programme. Whether implementation of fetal $R H D$ testing is cost effective in a screening programme depends on the characteristics of the test, economies of scale of testing, timing of testing in pregnancy, and costs of anti-D immunoglobulin, as described for the USA, ${ }^{30}$ Canada, ${ }^{31}$ and the UK. ${ }^{32}$ Fetal $R H D$ testing can be performed reliably from 11 weeks 
onwards, which would also facilitate the targeted use of anti-D immunoglobulin in certain clinical conditions, such as invasive procedures, late miscarriage, termination of pregnancy, abdominal trauma, or preterm birth. ${ }^{28} 29$ This has not yet been one of the aims of the Dutch antenatal screening programme. A further benefit of the use of fetal RHD testing may be direct use of anti-D immunoglobulin after delivery, preventing mistakes and delays. ${ }^{33}$ Three recent publications describe decision analytical models to evaluate the introduction of fetal RHD testing in a US population, ${ }^{34}$ Swedish population, ${ }^{35}$ and Canadian population. ${ }^{36}$ Two of the studies ${ }^{3435}$ point to an increased risk of RhD alloimmunisation owing to false negative fetal RHD test results. But neither of these studies took into account the putative errors of a procedure with cord blood testing or the benefits of fetal RHD testing for detection of weak RhD expression. Also, maternal alloimmunisation will not occur in all women with a false negative fetal $R H D$ result. The study in a Canadian population ${ }^{36}$ concluded that targeted anti-D immunoglobulin prophylaxis is both feasible and cost saving. These three studies illustrate that the estimated number of women at risk for $\mathrm{RhD}$ alloimmunisation depends on both the characteristics of the fetal $R H D$ test and the used risk for $\mathrm{RhD}$ alloimmunisation. Our study indicates that the false negative rate of fetal $R H D$ testing at 27 weeks can be regarded at least as low as in a setting with cord blood typing, and with acceptable numbers of false positive or inconclusive results. In agreement with another study, ${ }^{37}$ we regard the introduction of fetal RHD testing on the basis of targeted anti-D immunoglobulin use to be in line with the policy of reducing the unnecessary use of blood products. The percentage of Dutch women who received unnecessary anti-D immunoglobulin prophylaxis was reduced from about $40 \%$ (ie, in all $\mathrm{RhD}$ negative women carrying an $\mathrm{RhD}$ negative fetus) to $1.31 \%$ (ie, women with a false positive result and women who received prophylaxis despite a negative fetal $R H D$ test result). Our study showed that obstetric care providers comply with the programme and that pregnant women rarely refuse the test. This was also concluded in a qualitative study in the UK. ${ }^{38}$

\section{Strengths and limitations of this study}

A strength of the study is the large series of fetal $R H D$ test results obtained in pregnancy and compared with cord blood serology results. Tests were standardised and performed in a centralised setting. A thorough evaluation of false negative and false positive results was performed. Limitations of the study are that fetal $R H D$ testing was performed in pregnancy week 27 , whereas some countries perform the screening earlier in pregnancy to enable targeted anti-D immunoglobulin prophylaxis on putative sensitising events. More false negative results might, however, occur in the first trimester owing to low fetal DNA levels. Another limitation was the percentage of cases with a missing cord blood typing result. Our study, however, included almost the entire population rather than a selected sample.

\section{Conclusion and policy implications}

Fetal $R H D$ testing in week 27 of pregnancy is highly reliable and can be used as a single test to prevent unnecessary use of anti-D immunoglobulin during pregnancy in $\mathrm{RhD}$ negative women carrying an RhD negative child, and also to guide use of postnatal anti-D immunoglobulin. The number of false negative test results is at such a low level that fetal RHD testing on the basis of targeted use of anti-D immunoglobulin is not expected to increase the number of alloimmunised $\mathrm{RhD}$ negative women; in fact it might improve the logistical use of postnatal anti-D immunoglobulin. Cord blood serology is no longer performed in most pregnancies, but the accuracy of the screening programme is monitored indirectly through testing for anti-red blood cell antibodies in subsequent pregnancies. The compliance of the programme in the Netherlands was over $98 \%$, illustrating the acceptance of the test by obstetric care providers and pregnant women.

\section{AUTHOR AFFILIATIONS}

${ }^{1}$ Department of Experimental Immunohematology, Sanquin Research, Amsterdam and Landsteiner Laboratory, Academic Medical Center, University of Amsterdam, Amsterdam, Netherlands ${ }^{2}$ Center for Clinical Transfusion Research, Sanquin Research and Department of Immunohematology and Blood Transfusion, Leiden University Medical Center, Leiden

${ }^{3}$ Department of Immunohematology Diagnostic Services, Sanquin Research, Amsterdam, Netherlands

uUniversity Medical Center Utrecht, Division Woman and Baby, Department of Obstetrics, Utrecht, Netherlands

${ }^{5}$ Netherlands Organization for Applied Scientific Research, Department of Child Health, Leiden, Netherlands

${ }^{6}$ National Institute for Public Health and the Environment, Service for vaccine provision and prevention programs, Bilthoven, Netherlands

${ }^{7}$ National Institute for Public Health and the Environment, Center for population screening, Bilthoven, Netherlands

Contributors: MdH, FFT, Scheffer, and CEvdS conceived and designed the study. All authors acquired, analysed, or interpreted the data. MdH, FFT, PGS, CEvdS, and CPBvdP drafted the manuscript. All authors critically revised the manuscript for important intellectual content and gave final approval of the version to be published. MdH and FFT contributed equally to this manuscript. $\mathrm{MdH}$ is the guarantor.

Funding: This study received no specific funding.

Competing interests: All authors completed the ICMJE uniform disclosure form at www.icmje.org/coi_disclosure.pdf and declare: no support from any organisation for the submitted work; no financial relationships with any organisations that might have an interest in the submitted work in the previous three years; no other relationships or activities that could appear to have influenced the submitted work.

Ethical approval: Not required according to Dutch laws. This study was conducted according to the principles of the Declaration of Helsinki.

Data sharing: No additional data available.

Transparency: The manuscript's guarantor $(\mathrm{MdH})$ affirms that this manuscript is an honest, accurate, and transparent account of the study being reported; that no important aspects of the study have been omitted; and that any discrepancies from the study as planned have been explained.

This is an Open Access article distributed in accordance with the Creative Commons Attribution Non Commercial (CC BY-NC 3.0) license, which permits others to distribute, remix, adapt, build upon this work non-commercially, and license their derivative works on different terms, provided the original work is properly cited and the use is non-commercial. See: http://creativecommons.org/licenses/ by-nc/3.0/.

1 Urbaniak SJ, Greiss MA. RhD haemolytic disease of the fetus and the newborn. Blood Rev 2000;14:44-61. doi:10.1054/ blre.1999.0123. 
2 de Haas M, Thurik FF, Koelewijn JM, van der Schoot CE. Haemolytic disease of the fetus and newborn. Vox Sang 2015;109:99-113. doi:10.1111/vox.12265.

3 Crowther C, Middleton P. Anti-D administration after childbirth for preventing Rhesus alloimmunisation. Cochrane Database Syst Rev 2000;(2):CD000021.

4 Koelewijn JM, de Haas M, Vrijkotte TG, Bonsel GJ, van der Schoot CE. One single dose of 200 microg of antenatal RhIG halves the risk of anti-D immunization and hemolytic disease of the fetus and newborn in the next pregnancy. Transfusion 2008:48:1721-9. doi:10.1111/j.1537-2995.2008.01742.x

5 Pilgrim H, Lloyd-Jones M, Rees A. Routine antenatal anti-D prophylaxis for RhD-negative women: a systematic review and economic evaluation. Health Technol Assess 2009;13(10):iii,ix-xi,1-103.

6 Crowther CA, Keirse MJ. Anti-D administration in pregnancy for preventing rhesus alloimmunisation. Cochrane Database Syst Rev 2000;(2):CD000020.

7 Daniels G. Human blood groups. Wiley Online Library, 2013: 185. doi:10.1002/9781118493595

8 Lo YM, Corbetta N, Chamberlain PF, et al. Presence of fetal DNA in maternal plasma and serum. Lancet 1997;350:485-7. doi:10.1016/ S0140-6736(97)02174-0

9 Faas BH, Beuling EA, Christiaens GC, von dem Borne AE, van der Schoot CE. Detection of fetal RHD-specific sequences in maternal plasma. Lancet 1998;352:1196. doi:10.1016/ S0140-6736(05)60534-X.

10 Lo YM, Hjelm NM, Fidler C, et al. Prenatal diagnosis of fetal RhD status by molecular analysis of maternal plasma. N Engl J Med 1998;339:1734-8. doi:10.1056/NEJM199812103392402.

11 Bianchi DW, Avent ND, Costa JM, van der Schoot CE. Noninvasive prenatal diagnosis of fetal Rhesus D: ready for Prime(r) Time. Obstet Gynecol 2005;106:841-4. doi:10.1097/01. AOG.0000179477.59385.93.

12 Van der Schoot CE, Soussan AA, Koelewijn J, Bonsel G, PagetChristiaens LG, de Haas M. Non-invasive antenatal RHD typing Transfus Clin Biol 2006;13:53-7. doi:10.1016/j.tracli.2006.02.021.

13 Müller SP, Bartels I, Stein W, et al. The determination of the fetal D status from maternal plasma for decision making on Rh prophylaxis is feasible. Transfusion 2008;48:2292-301. doi:10.1111/j.1537-2995.2008.01843.x.

14 Finning K, Martin P, Summers J, Massey E, Poole G, Daniels G. Effect of high throughput RHD typing of fetal DNA in maternal plasma on use of anti-RhD immunoglobulin in RhD negative pregnant women: prospective feasibility study. BMJ 2008;336:816-8. doi:10.1136/ bmj.39518.463206.25.

15 Avent ND, Reid ME. The Rh blood group system: a review. Blood 2000:95:375-87.

16 Grootkerk-Tax MG, Soussan AA, de Haas M, Maaskant-van Wijk PA van der Schoot CE. Evaluation of prenatal RHD typing strategies on cell-free fetal DNA from maternal plasma. Transfusion 2006;46:2142 8. doi:10.1111/j.1537-2995.2006.01044.x.

17 Clausen FB, Christiansen M, Steffensen R, et al. Report of the first nationally implemented clinical routine screening for fetal $R H D$ in D- pregnant women to ascertain the requirement for antenatal RhD prophylaxis. Transfusion 2012:52.752-8 doi:10.1111/j.1537-2995.2011.03362.x

18 Health Council of the Netherlands. Pregnancy immunisation by red blood cells. The Hague: Health Council of the Netherlands, 2009; publication No 2009/04. ISBN 978-90-5549-753-9.

19 Haer-Wigman L, Veldhuisen B, Jonkers R, et al. RHD and RHCE variant and zygosity genotyping via multiplex ligation-dependent probe amplification. Transfusion 2013;53:1559-74 doi:10.1111/j.1537-2995.2012.03919.x

20 Legler TJ, Liu Z, Mavrou A, et al. Workshop report on the extraction of foetal DNA from maternal plasma. Prenat Diagn 2007;27:824-9. doi:10.1002/pd.1783.

21 Scheffer PG, van der Schoot CE, Page-Christiaens GC, de Haas M. Noninvasive fetal blood group genotyping of rhesus D, c, E and of $\mathrm{K}$ in alloimmunised pregnant women: evaluation of a 7-year clinical experience. BJOG 2011;118:1340-8. doi:10.1111/j.1471-0528.2011.03028.x
22 Stegmann TC, Veldhuisen B, Bijman R, et al. Frequency and characterization of known and novel RHD variant alleles in 37782 Dutch D-negative pregnant women. Br J Haematol 2016;173:469-79. doi:10.1111/bjh.13960

23 Fleiss JL. Statistical Methods for Rates and Proportions. 2nd edn. John Wiley \& Sons, London, 1981.

24 Thurik FF, Ait Soussan A, Bossers B, et al. Analysis of false-positive results of fetal RHD typing in a national screening program reveals vanishing twins as potential cause for discrepancy. Prenat Diagn 2015:35:754-60. doi:10.1002/pd.4600.

25 van der Ploeg CPB, Schönbeck Y, Hirschberg H. Prenatale Screening Infectieziekten en Erytrocytenimmunisatie (PSIE). Procesmonitor 2012; RIVM/TNO 2014. http://repository.tudelft.nl/view/tno/ uuid\%3Af3c09fc0-bb5d-4f34-9daa-cb4cec699090/. Accessed January 20, 2016.

26 Legler TJ, Müller SP, Haverkamp A, Grill S, Hahn S. Prenatal RhD Testing: A Review of Studies Published from 2006 to 2008. Transfus Med Hemother 2009;36:189-98. doi:10.1159/000216580.

27 Clausen FB, Steffensen R, Christiansen M, et al. Routine noninvasive prenatal screening for fetal RHD in plasma of RhD-negative pregnant women-2 years of screening experience from Denmark. Prenat Diagn 2014;34:1000-5. doi:10.1002/pd.4419.

28 Chitty LS, Finning K, Wade A, et al. Diagnostic accuracy of routine antenatal determination of fetal RHD status across gestation: population based cohort study. BMJ 2014;349:g5243. doi:10.1136/ bmj.g5243.

29 Wikman AT, Tiblad E, Karlsson A, Olsson ML, Westgren M, Reilly M. Noninvasive single-exon fetal $R H D$ determination in a routine screening program in early pregnancy. Obstet Gynecol 2012;120:22734. doi:10.1097/AOG.0b013e31825d33d9.

30 Hawk AF, Chang EY, Shields SM, Simpson KN. Costs and clinical outcomes of noninvasive fetal RhD typing for targeted prophylaxis. Obstet Gynecol 2013;122:579-85. doi:10.1097/ AOG.0b013e31829f8814.

31 Duplantie J, Martinez Gonzales O, Bois A, et al. Cost-effectiveness of the management of rh-negative pregnant women. J Obstet Gynaecol Can 2013;35:730-40. doi:10.1016/S1701-2163(15)30864-1.

32 Szczepura A, Osipenko L, Freeman K. A new fetal RHD genotyping test: costs and benefits of mass testing to target antenatal anti-D prophylaxis in England and Wales. BMC Pregnancy Childbirth 2011:11:5. doi:10.1186/1471-2393-11-5.

33 Bolton-Maggs PH, Davies T, Poles D, Cohen H. Errors in anti-D immunoglobulin administration: retrospective analysis of 15 years of reports to the UK confidential haemovigilance scheme. BJOG 2013;120:873-8. doi:10.1111/1471-0528.12175.

34 Ma KK, Rodriguez MI, Cheng YW, Norton ME, Caughey AB. Should cell-free DNA testing be used to target antenatal rhesus immune globulin administration?] Matern Fetal Neonatal Med 2016;29:1866-70.

35 Neovius M, Tiblad E, Westgren M, et al. Cost $\square$ effectiveness of first trimester nonDinvasive fetal RHD screening for targeted antenatal anti $\square$ p prophylaxis in RhD $\square$ negative pregnant women: a model $\square$ based analysis. BJOG 2016;123:1337-46. doi:10.1111/1471-0528.13801.

36 Teitelbaum L, Metcalfe A, Clarke G, Parboosingh JS, Wilson RD, Johnson JM. Costs and benefits of non-invasive fetal RhD determination. Ultrasound Obstet Gynecol 2015;45:84-8. doi:10.1002/uog.14723.

37 Kent J, Farrell AM, Soothill P. Routine administration of Anti-D: the ethical case for offering pregnant women fetal RHD genotyping and a review of policy and practice. BMC Pregnancy Childbirth 2014;14:87. doi:10.1186/1471-2393-14-87

38 Oxenford K, Silcock C, Hill M, Chitty L. Routine testing of fetal Rhesus D status in Rhesus D negative women using cell-free fetal DNA: an investigation into the preferences and information needs of women. Prenat Diagn 2013;33:688-94. doi:10.1002/pd.4135.

Supplementary file: supplementary eTables 1-4 and eFigure 1 